First dental students practise dentistry from home

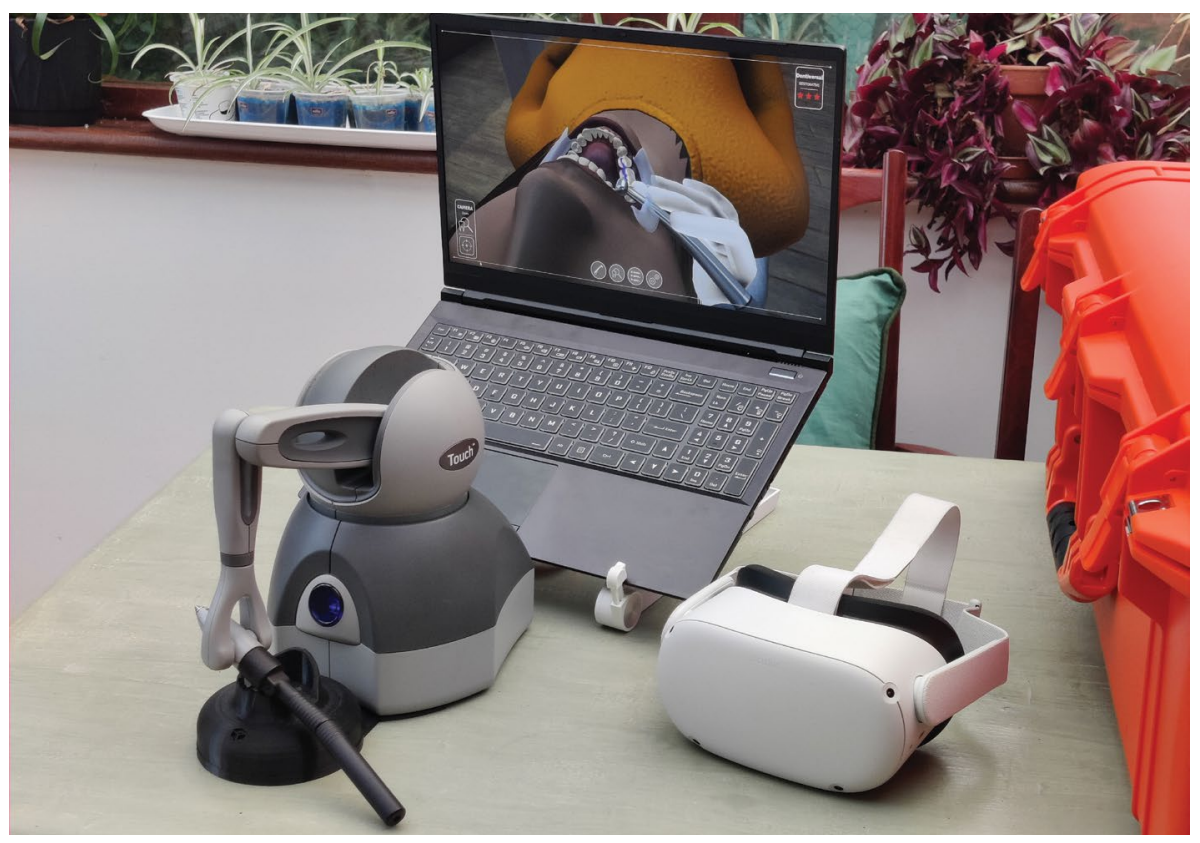

Uni Sim (Universal Simulation UK) has launched the world's first portable dental training simulator for dentists, using virtual reality and haptics. A laptop and 'haptic' device allows dentists to practise treatments virtually and to really feel the different sensations of drilling through enamel and dentine. Much in the same way that flight simulators have permitted pilots to practise flying - without the need (and risk!) of practising with a real plane - this is allowing students to practise without a real patient from the comfort of their home.

It is being viewed as an opportunity to continue student education remotely during periods of uncertainty regarding lockdowns, quarantines and student access. Following external use, it can be brought back into the university and integrated into the simulation suite.

The first devices are being used at ADEMA University, in Spain, that are allowing students to practise from home during quarantine. It is also allowing the university to develop the world's first remote dental education course using haptics.

Uni Sim are targeting dental operations to begin with, starting with simulations in restoration, endodontics, prosthodontics and oral surgery. They are also developing a simulation to teach anaesthesia in a project with New York University and ADEMA University.

You can find out more at www.unisim. co.uk oremail contact@unisim.co.uk.

\section{Shock treatment for dental unit waterlines launched}

A-dec are pleased to announce the launch of ICX Renew, a shock treatment specifically designed to penetrate and remove the build-up of odour and foultasting microbial contamination from dental unit waterlines.

Unwanted visitors like bacteria love to colonise in your dental unit waterlines, along with a daily waterline maintenance protocol such as A-dec ICX, it's important to monitor water quality and periodically shock the lines to clear deposits.

\section{A-dec ICX and ICX Renew help} simplify your waterline maintenance routine. Ready to use and formulated without harsh chemicals, treating dental unit waterlines with ICX products help protect your investment.

Claim your special introductory discount code at a-dec.com/shockingoffer (terms and conditions apply).

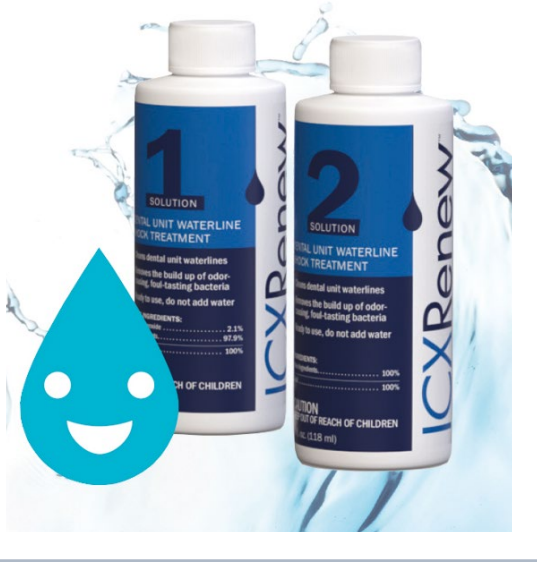

\title{
Remember the colour code
}

Does your team sometimes find it hard to remember which waste belongs where?

Help ensure they never make a mistake during waste disposal with Colour Code Character Posters from Initial Medical.

Personifications of each waste stream, these fun, vibrant characters make learning the Department of Health's colour coded guidelines for best practice waste disposal easy and stress-free. By attaching visual aids as well as the colour to each waste stream, people are likely to remember the correct disposal method for each type of waste and what items are classified under each umbrella.
Plus, these posters are free to download here: https://www.initial.co.uk/ colour-coding-guide/.

Make waste disposal easy today by contacting the team at Initial Medical.

For further information visit www. initial.co.uk/medical or Tel: 08708504045 .

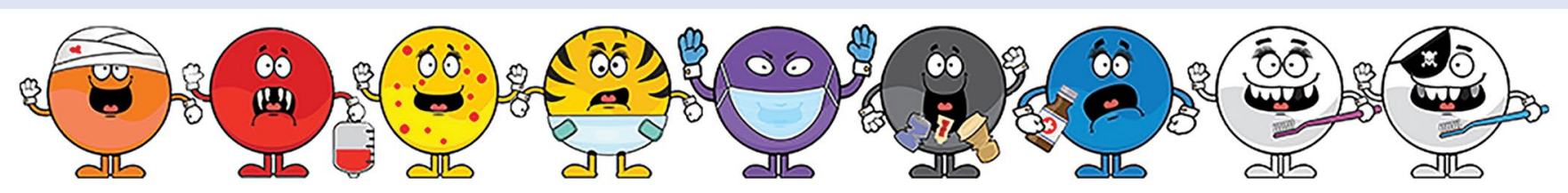

